\title{
Associações entre as biomassas de peixes Sciaenidae (Teleostei: Perciformes) e de camarões Penaeoidea (Decapoda: Dendrobranchiata) no litoral norte do Estado de São Paulo
}

\author{
Ursulla Pereira Souza ${ }^{1,6}$; Rogério Caetano da Costa $^{2,5}$; Itamar Alves Martins ${ }^{3}$; Adilson Fransozo ${ }^{4,5}$ \\ ${ }^{1}$ Departamento de Zoologia, Instituto de Biociências, Universidade Estadual Paulista - UNESP, \\ Av. 24a, $n^{\circ}$ 1515, CP 199, CEP 13506-900, Rio Claro, SP, Brasil \\ ${ }^{2}$ Departamento de Ciências Biológicas, Faculdade de Ciências, Universidade Estadual Paulista - UNESP, \\ Av. Eng. Edmundo Carrijo Coube, $n^{\circ}$ 14-01, CEP 17033-360, Bauru, SP, Brasil, e-mail: rccosta@fc.unesp.br \\ ${ }^{3}$ Laboratório de Zoologia, Instituto Básico de Biociências, Universidade de Taubaté - UNITAU, \\ Av. Tiradentes, $n^{\circ}$ 500, CEP 12030-180,Taubaté, SP, Brasil, e-mail: istama@uol.com.br \\ ${ }^{4}$ Departamento de Zoologia, Universidade Estadual Paulista - UNESP, \\ Distrito de Rubião Jr., s/n ${ }^{o}$, CEP 18618-000, Botucatu, SP, Brasil, e-mail: fransozo@ibb.unesp.br, \\ ${ }^{5}$ Núcleo de Estudos em Biologia, Ecologia e Cultivo de Crustáceos - NEBECC \\ ${ }^{6}$ Autor para correspondência: Ursulla Pereira Souza, e-mail: upsouza@rc.unesp.br
}

Souza, U. P.; Costa, R. C.; Martins, I. A.; Fransozo, A. Relationships among Sciaenidae fish (Teleostei: Perciformes) and Penaeoidea shrimp (Decapoda: Dendrobranchiata) biomass from the north coast of São Paulo State, Brazil. Biota Neotrop., vol 8, no. 1, Jan./Mar. 2008. Available from: <http://www.biotaneotropica. org.br/v8n1/en/abstract?article+bn02108012008>.

\begin{abstract}
To study the relationships among Sciaenidae fish and Penaeoidea shrimp biomass, fieldwork was carried out in the north coast of São Paulo state, Brazil, during the four seasons of 2001. Samples were collected in transects from different depths at Ubatuba, Caraguatatuba, and São Sebastião cities. Water and sediment were collected from each transect. The hypotheses that the environmental factors were different among the seasons and depths were tested by ANOVAs. The statistical dependence among the biomasses of fishes (response variable) and the biomasses of shrimps (covariable) in the three sections was tested by an ANCOVA model. CCA was used to quantify the relationships among each species' biomass. The seasons and sections important at the CCA results were evaluated by Kruskal-Wallis test and depth by Spearman's test. The temperatures and salinities were significantly different among seasons but organic matter and phi were not. A total of 378,618 $\mathrm{g}$ of fish and 79,338 $\mathrm{g}$ of shrimp were collected and the majority was captured during the winter and in shallow transects. The greatest biomass of both groups was captured at Caraguatatuba and the correlation between fish and shrimp captures was negative. A positive correlation was found in the other areas. Therefore, data from Caraguatatuba was removed from the final ANCOVA model, in which $47 \%$ of Sciaenidae biomass variability was explained by the presence of Penaeoidea shrimps. Xiphopenaeus kroyeri was the only important factor in Sciaenidae distribution in the axis 1 , while axis 2 indirectly evidenced the depth gradient. The results suggest that many fish species search for the same areas where shrimp occurs, related to abiotic factors, and use them as food resource.
\end{abstract}

Keywords: sciaenids, penaeids, Ubatuba, Caraguatatuba, São Sebastião, ANCOVA.

Souza, U. P.; Costa, R. C.; Martins, I. A.; Fransozo, A. Associações entre as biomassas de peixes Sciaenidae (Teleostei: Perciformes) e de camarões Penaeoidea (Decapoda: Dendrobranchiata) no litoral norte do Estado de São Paulo. Biota Neotrop., vol 8, no. 1, jan./mar. 2008. Disponível em: <http://www.biotaneotropica.org.br/ v8n1/pt/abstract?article+bn02108012008>.

Resumo: Visando analisar as associações entre as biomassas de peixes Sciaenidae e de camarões Penaeoidea, foram feitas amostragens com barco de arrasto nas quatro estações do ano de 2001, em um gradiente de profundidade, nas regiões de Ubatuba, Caraguatatuba e São Sebastião. Em cada transecto, foram obtidas amostras de água e de sedimento. As hipóteses de que os fatores ambientais foram diferentes entre as estações do ano e profundidades foram testadas por ANOVAs. A dependência estatística entre as biomassas de peixes (variável resposta) e as biomassas de camarões (covariável) nas três regiões, foi testada por um modelo de ANCOVA. Para quantificar as relações entre as biomassas de cada espécie foi utilizada a CCA. A importância das estações do ano e das regiões no resultado da CCA foi avaliada pelo teste de Kruskal-Wallis e da profundidade pelo teste de Spearman. Nas estações do ano, as temperaturas e salinidades foram significativamente diferentes, o que não ocorreu com matéria orgânica (MO) e o diâmetro médio do sedimento (phi $=\Phi$ ). Foram amostrados $378.618 \mathrm{~g}$ de peixes e $79.338 \mathrm{~g}$ de camarões, com maiores capturas no inverno e nas menores profundidades. Em Caraguatatuba foram coletadas as maiores biomassas de ambos os grupos e a correlação entre as capturas de peixes e de camarões foi negativa. Já nas demais regiões, a correlação foi positiva. Assim, os dados de Caraguatatuba foram retirados do modelo final da ANCOVA, onde $47 \%$ da variabilidade na biomassa dos peixes Sciaenidae pôde ser explicada pela presença dos camarões Penaeoidea. Xiphopenaeus kroyeri foi o único fator importante na distribuição de Sciaenidae no eixo 1, enquanto o eixo 2 evidenciou indiretamente o gradiente de profundidade. Os resultados sugerem que, possivelmente, muitas espécies de peixes busquem as mesmas áreas de ocorrência dos camarões em função dos fatores abióticos ou para utilizá-los como recurso alimentar.

Palavras-chave: cienídeos, peneídeos, Ubatuba, Caraguatatuba, São Sebastião, ANCOVA. 


\section{Introdução}

O ambiente costeiro desempenha um papel essencial no desenvolvimento de larvas e jovens de peixes, constituindo regiões relativamente ricas em alimento, que podem funcionar como criadouros naturais para muitas espécies (Braga \& Goitein 1984). Os peixes da família Sciaenidae são mais comumente encontrados em águas rasas da plataforma continental, sobre fundos de areia ou lama (Menezes \& Figueiredo 1980) e constituem o mais importante recurso pesqueiro em águas costeiras e estuarinas do mundo (Chao 1986), sendo representados no Brasil por 21 gêneros e cerca de 54 espécies marinhas (Menezes et al. 2003). As espécies apresentam diferentes modos de alimentação, relacionados a variações nas características morfológicas e padrões de história de vida (Chao \& Musick 1977) e muitas utilizam o estuário como berçário e local de alimentação para os jovens (Chao 1986).

As espécies de Sciaenidae consideradas mais abundantes na região sudeste do Brasil, nas proximidades de Ubatuba, são Ctenosciaena gracilicirrhus, Paralonchurus brasiliensis e Cynoscion jamaicensis (Cunningham 1983, Nonato et al. 1983, Braga \& Goitein 1984, Rocha 1990, Micheletti \& Uieda 1996, Rocha \& Rossi-Wongtschowski 1998) e, na região sul Umbrina canosai, Cynoscion guatucupa (= C. striatus), Micropogonias furnieri e Macrodon ancylodon (Vazzoler 1975, Haimovici et al. 1989).

A maioria dos Sciaenidae ocorre como fauna acompanhante da pesca dos camarões (Rodrigues \& Meira 1988). Na costa do Brasil existem sete famílias de camarões Dendrobranchiata, representadas por 26 gêneros e 61 espécies (Costa et al. 2000), sendo que no sudeste, a pesca é direcionada aos camarões da família Penaeidae, principalmente aos camarões-rosa (Farfantepenaeus brasiliensis e F. paulensis), branco (Litopenaeus schmitti) e sete-barbas (Xiphopenaeus kroyeri) (D'Incao et al. 2002).

De acordo com Sheridan et al. (1984), Sciaenidae, Haemulidae, Sparidae, Synodontidae, Serranidae e Paralichthyidae são as famílias de peixes demersais comumente encontradas nas mesmas áreas de ocorrência dos camarões Penaeidae, sendo que a determinação da natureza e extensão das interações ecológicas entre peixes e camarões é importante para a manutenção e exploração racional de ambos os estoques pesqueiros. Os autores ressaltam, ainda, a escassez de estudos quantitativos que permitam dimensionar adequadamente a importância dos camarões nas relações tróficas destes peixes demersais.

Alguns estudos têm sugerido fortes associações entre peixes e camarões por meio da predação (Sheridan et al. 1984, Dall et al. 1990, Brewer et al. 1995, Nakagaki 1999, Macia et al. 2003) e outros demonstraram que peixes Sciaenidae utilizam os camarões Penaeoidea como principal recurso alimentar (Rodrigues \& Meira 1988, Brewer et al. 1995, Micheletti \& Uieda 1996, Jucá-Chagas 1997, Soares \& Vazzoler 2001).

Dall et al. (1990) destacaram que a predação dos peixes sobre os camarões, em especial a exercida pelos Sciaenidae, atinge intensidades três vezes maiores que a da frota pesqueira, havendo uma variação entre as diversas fases do ciclo de vida dos Penaeidae, com as taxas mais elevadas verificadas sobre as formas larvais destes camarões. Nakagaki (1999) ressalta que estudos da contribuição dos camarões para níveis tróficos superiores podem ser importantes para um melhor entendimento da dinâmica populacional do grupo.

A variabilidade na composição da dieta de uma dada espécie, embora relacionada ao seu comportamento e à sua morfologia, está fortemente associada a diferenças na abundância local do alimento (Jucá-Chagas 1997). Assim, o caráter sazonal da disponibilidade do recurso alimentar pode ser o fator chave que afeta vários aspectos da dinâmica da comunidade (Lowe-McConnell 1999).
Embora alguns estudos sugiram a dependência entre as abundâncias de peixes Sciaenidae e de camarões Penaeoidea, não existem indicações de como esta relação varia temporal e espacialmente. Assim, este estudo visou analisar a relação entre as biomassas de peixes Sciaenidae e de camarões Penaeoidea, ao longo de um gradiente de profundidade e nas estações do ano de 2001, em três regiões do litoral norte do estado de São Paulo. Também foram avaliadas as variações espaciais e temporais de alguns fatores abióticos importantes nas distribuições destas espécies.

\section{Material e Métodos}

As amostragens foram realizadas nas quatro estações do ano de 2001 (verão, outono, inverno e primavera), nas regiões de Ubatuba

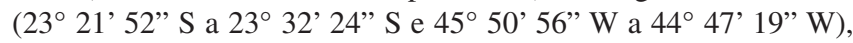
Caraguatatuba (23 $37^{\prime} 53^{\prime \prime} \mathrm{S}$ a $23^{\circ} 44^{\prime} 16^{\prime \prime} \mathrm{S}$ e $45^{\circ} 00^{\prime} 22^{\prime \prime} \mathrm{W}$ a $45^{\circ} 03^{\prime} 02^{\prime \prime}$ W) e São Sebastião (23 $51^{\prime} 36^{\prime \prime}$ S a $23^{\circ} 58^{\prime} 10^{\prime \prime} \mathrm{S}$ e $45^{\circ} 30^{\prime} 30^{\prime \prime} \mathrm{W}$ a $45^{\circ} 29^{\prime} 44^{\prime \prime} \mathrm{W}$ ), efetuadas em transectos localizados nas profundidades de 5, 15, 25, 35 e $45 \mathrm{~m}$ (Figura 1).

As coletas foram diurnas utilizando-se um barco de pesca comercial, equipado com duas redes de arrasto do tipo "double-rig", com 4,5 m de abertura e $11 \mathrm{~m}$ de comprimento. A distância entre-nós, na panagem da rede e no saco final, foram de 20 e $15 \mathrm{~mm}$, respectivamente. Em cada transecto foram percorridos, aproximadamente, $2 \mathrm{~km}$ em 30 minutos.

Os peixes e os camarões capturados foram pesados e identificados ao nível de espécie de acordo com os trabalhos de Menezes \& Figueiredo (1980) e Cervigón et al. (1992), para os Sciaenidae e de Pérez Farfante \& Kensley (1997) e Costa et al. (2003), para os Penaeoidea. Alguns exemplares de peixes foram depositados na Coleção Científica de Ictiologia do departamento de Zoologia da Universidade de Taubaté (IAM/CCILZU) e de camarões no departamento de Zoologia da Universidade Estadual Paulista, campus de Botucatu, SP.

Nos transectos foram coletadas amostras de água de superfície e de fundo com uma garrafa de Nansen e mensuradas a temperatura (com um termômetro) e a salinidade (com um refratômetro óptico específico). As amostras de sedimento foram obtidas com o pegador do tipo van Veen (área de $1 / 40 \mathrm{~m}^{2}$ ), a partir das quais foram calculados o teor de matéria orgânica (MO) e o diâmetro médio do sedimento (phi = Ф), segundo Hakanson \& Jansson (1983) e Tucker (1988).

Para testar as hipóteses de que alguns fatores ambientais foram diferentes entre as estações do ano e profundidades amostradas, foram utilizados modelos de análise de variância (ANOVAs two-way), tendo como variáveis dependentes as temperaturas (superfície e fundo), as salinidades (superfície e fundo), o teor de matéria orgânica e o diâmetro médio do sedimento. Os teores de matéria orgânica (MO) foram transformados $(\ln (x+1))$ para atender as premissas da análise. A normalidade dos resíduos foi verificada pelo Teste de Lilliefors e a homogeneidade de variâncias pelo Teste de Leveane (Zar 1999). Quando algum desses pressupostos não foi satisfeito, as ANOVAs foram substituídas pelo teste não-paramétrico de Kruskal-Wallis.

A hipótese de dependência entre as biomassas dos peixes e dos camarões nas três regiões foi avaliada por um modelo de análise de covariância (ANCOVA), tendo como variável resposta o logaritmo natural (ln) da biomassa dos peixes, como covariável o logaritmo natural (ln) da biomassa dos camarões e como fatores, as regiões. Foi estabelecido o nível de significância $(\alpha)$ de $5 \%$.

As relações entre as biomassas de todas as espécies de peixes e de camarões que contribuíram com mais de 3\% do total capturado foram quantificadas pela análise de correspondência canônica (CCA, Ter Braak 1986). Este procedimento foi realizado no software R - versão 2.4.1 (R Development Core Team 2007), utilizando a função CCA disponível no pacote Vegan (Oksanen et al. 2006). A 


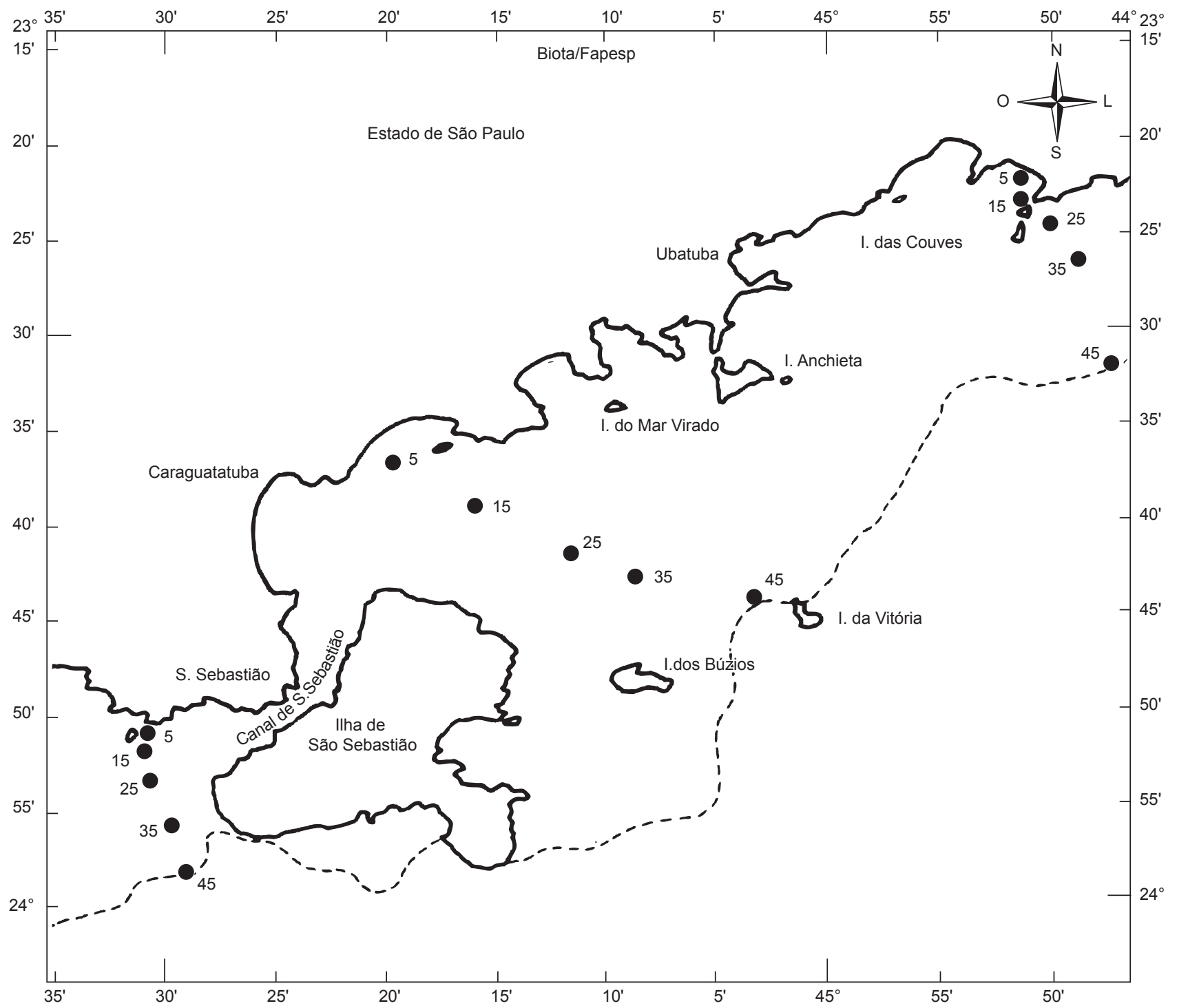

Figura 1. Mapa com a localização das profundidades nas regiões estudadas em Ubatuba, Caraguatatuba e São Sebastião.

Figure 1. Study area map with depth location at Ubatuba, Caraguatatuba and São Sebastião.

hipótese de que cada espécie de camarão apresente uma correlação maior que a esperada ao acaso com cada eixo foi verificada com a função "envfit". Assim, obtendo-se o valor de $\mathrm{r}^{2}$, a probabilidade de significância destas correlações $(p<0,05)$ foi calculada após 4999 permutações da matriz de biomassa de camarão. Nesta matriz as espécies pertencentes ao gênero Farfantepenaeus spp. (F. brasiliensis e F. paulensis) foram agrupadas, por apresentarem características semelhantes (Nakagaki 1999). Somente os dois primeiros eixos da CCA foram mantidos para interpretação. A importância das estações do ano e das regiões no resultado da CCA foi avaliada pelo teste de Kruskal-Wallis para cada um dos eixos, enquanto que o papel da profundidade, pelo teste de correlação de Spearman. As espécies de camarões, significativamente associadas a pelo menos um dos eixos, foram relacionadas individualmente às espécies de peixes pelo teste de Spearman.

\section{Resultados}

Todas as variáveis abióticas, exceto phi $(\Phi)$, atingiram os pressupostos de normalidade e homogeneidade de variâncias. Os resultados das ANOVAs efetuadas entre as variáveis abióticas, as estações do ano e as profundidades podem ser verificados na Tabela 1. As temperaturas (superfície e fundo) foram significativamente diferentes entre as estações, sendo verificado um maior valor na superfície durante o verão e o menor no fundo, durante a primavera (Figura 2a). Somente a temperatura de fundo apresentou relação com o gradiente de profundidade, sendo menor nos transectos mais fundos $(p<0,05)$ (Figura 2b). As salinidades (superfície e fundo) também estiveram significativamente relacionadas com as estações do ano, sendo mais elevadas no inverno e na primavera (Figura 3a), enquanto que nas profundidades não foram verificadas diferenças significativas (Figura 3b). Os teores de matéria orgânica (MO) não apresentaram diferenças significativas temporal ou espacialmente (Figura 4a, b).

Pelo resultado dos testes de Kruskal-Wallis, verificou-se que o diâmetro médio do sedimento não diferiu entre as estações do ano $(\mathrm{p}=0,957)$ (Figura $4 \mathrm{a})$. Nas profundidades amostradas houve diferença significativa $(\mathrm{p}<0,01)$, sendo os valores mais elevados nos transectos rasos (Figura $4 \mathrm{~b}$ ), caracterizando assim sedimentos mais finos. 
Souza, U. P. et al
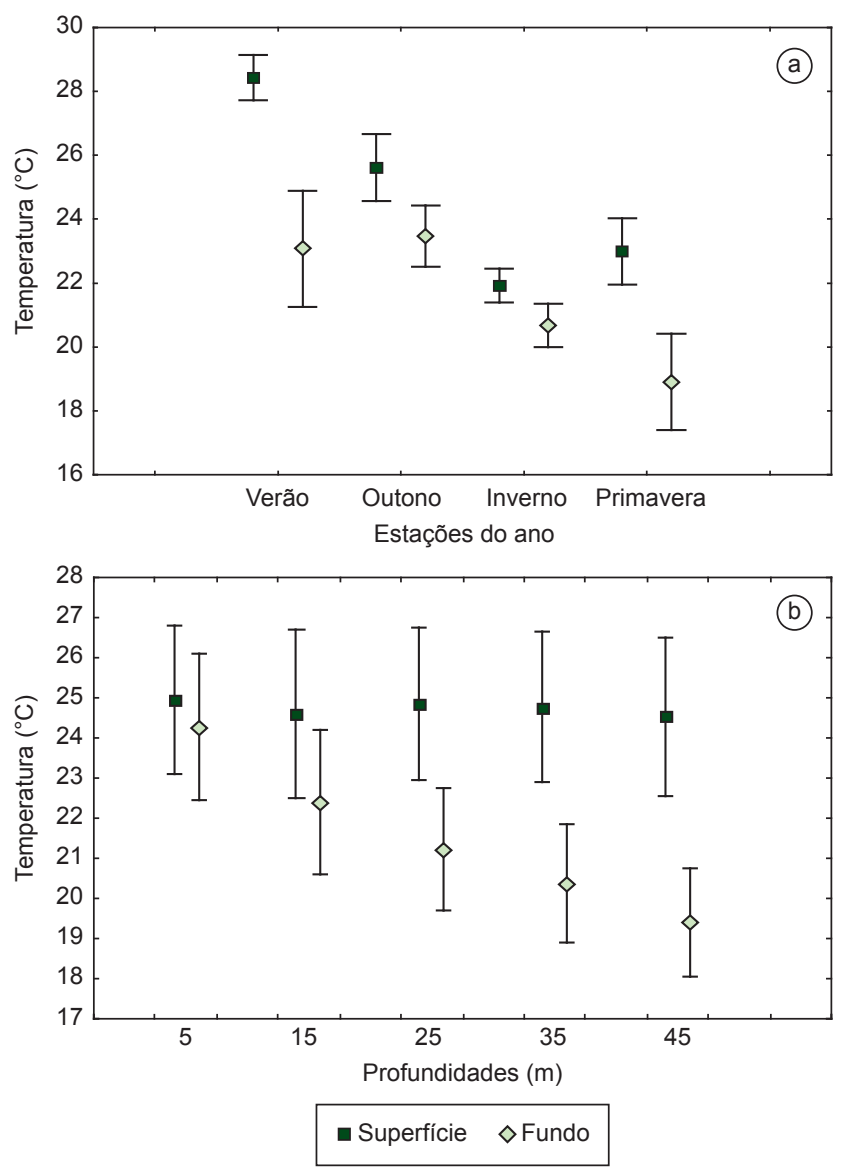

Figura 2. Média e intervalo de confiança (95\%) das temperaturas (superfície e fundo) a) nas estações do ano de $2001 \mathrm{e} \mathrm{b}$ ) em diferentes profundidades.

Figure 2. Mean temperature (surface and bottom) and confidence interval (95\%) at a) 2001 seasons and at b) different depths.

Tabela 1. Resultado das Análises de variância (ANOVAs) para os fatores ambientais (temperatura de superfície e de fundo, salinidade de superfície e de fundo e porcentagem de matéria orgânica), considerando as estações do ano e as profundidades amostradas em Ubatuba, Caraguatatuba e São Sebastião, durante o ano 2001.

Table 1. Analysis of variance (ANOVAs) results for environmental factors (surface and bottom temperature, surface and bottom salinity, and organic matter percentage), considering seasons and depths sampled at Ubatuba, Caraguatatuba and São Sebastião, during 2001.

\begin{tabular}{llrr}
\hline Variáveis dependentes & \multicolumn{1}{c}{ Fontes de variação } & \multicolumn{1}{c}{ F } & \multicolumn{1}{c}{ P } \\
\hline Temperatura (superfície) & Estações & 38,838 & $<\mathbf{0 , 0 1 0}$ \\
& Profundidades & 0,116 & 0,976 \\
& Estações * Profundidades & 0,079 & 1,000 \\
Temperatura (fundo) & Estações & 30,183 & $<\mathbf{0 , 0 1 0}$ \\
& Profundidades & 18,859 & $<\mathbf{0 , 0 1 0}$ \\
& Estações * Profundidades & 1,942 & 0,058 \\
Salinidade (superfície) & Estações & 12,185 & $<\mathbf{0 , 0 1 0}$ \\
& Profundidades & 0,123 & 0,973 \\
& Estações * Profundidades & 0,144 & 1,000 \\
Salinidade (fundo) & Estações & 5,664 & $\mathbf{0 , 0 0 2}$ \\
& Profundidades & 0,179 & 0,948 \\
& Estações * Profundidades & 0,124 & 1,000 \\
Matéria orgânica (MO) & Estações & 2,064 & 0,120 \\
& Profundidades & 1,085 & 0,377 \\
& Estações * Profundidades & 0,541 & 0,875 \\
\hline
\end{tabular}
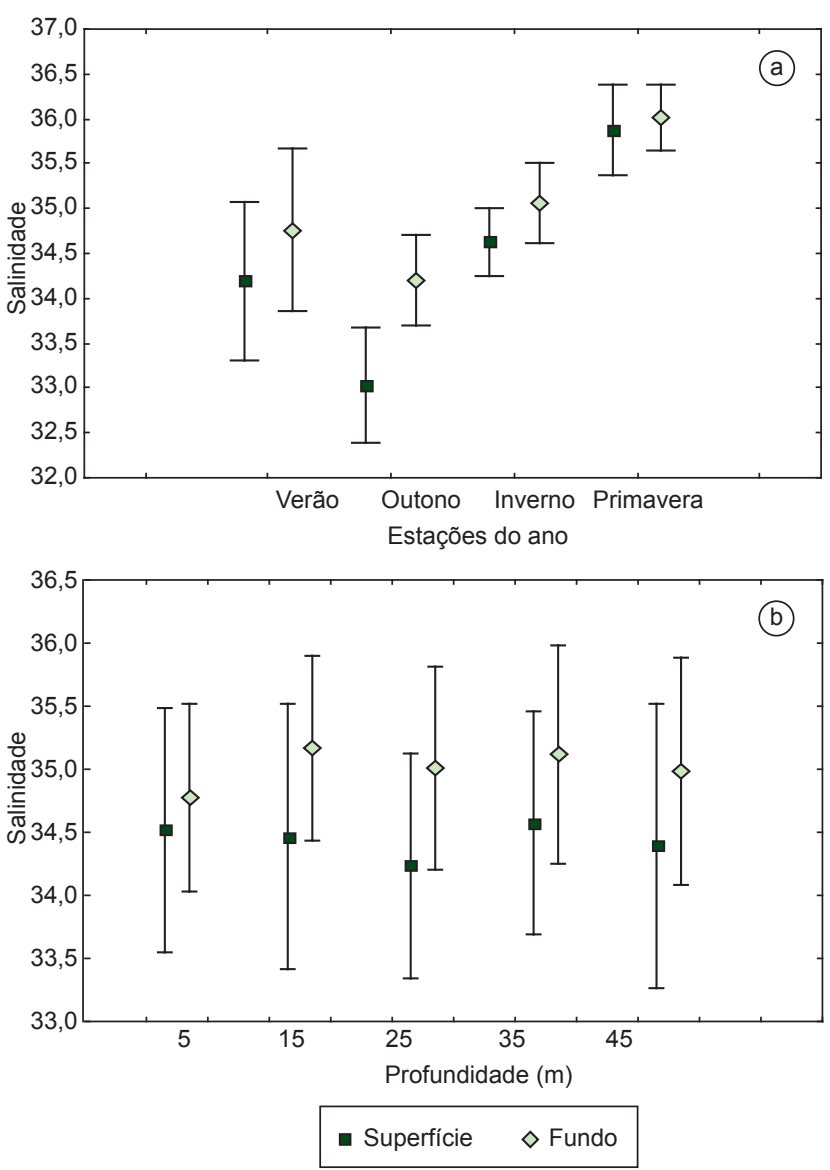

Figura 3. Média e intervalo de confiança (95\%) das salinidades (superfície e fundo) a) nas estações do ano de $2001 \mathrm{e} \mathrm{b}$ ) em diferentes profundidades.

Figure 3. Mean salinity (surface and bottom) and confidence interval (95\%) a) at 2001 seasons and b) at different depths.

Foram amostrados $378.618 \mathrm{~g}$ de peixes da família Sciaenidae, compreendendo 12 gêneros e 17 espécies e $79.338 \mathrm{~g}$ de camarões pertencentes à superfamília Penaeoidea, representados por três famílias, sete gêneros e nove espécies (Tabela 2).

Na região de Caraguatatuba foi amostrada a maior biomassa de peixes (161.710 g, 43\%), seguida por Ubatuba (121.700 g, 32\%) e São Sebastião (95.208 g, 25\%). Os camarões foram mais representativos também na região de Caraguatatuba (49.688g, 62\%), seguido por São Sebastião (15.844 g, 20\%) e Ubatuba (13.806 g, 18\%). As maiores amostragens de peixes e de camarões ocorreram no inverno e, a maior biomassa de peixes foi coletada na profundidade $5 \mathrm{~m}$ e a de camarões em $15 \mathrm{~m}$.

Paralonchurus brasiliensis, Ctenosciaena gracilicirrhus, Micropogonias furnieri, Menticirrhus americanus e Larimus breviceps contribuíram com $73 \%$ do peso total amostrado. Em Ubatuba a principal espécie capturada foi L. breviceps $(23 \%)$ e em Caraguatatuba e São Sebastião foi P. brasiliensis (25\% e 28\%, respectivamente). Os camarões Xiphopenaeus kroyeri e Pleoticus muelleri representaram $81 \%$ da biomassa de camarões, sendo as principais espécies amostradas nas três regiões.

Um modelo preliminar de análise de covariância (ANCOVA) resultou na interação significativa entre as regiões e a covariável $(\mathrm{p}=0,002)$. Plotando o logaritmo natural da biomassa de peixes versus a de camarões para cada região (Figura 5a, b, c), o padrão positivo esperado não foi observado em Caraguatatuba, onde existe uma corre- 

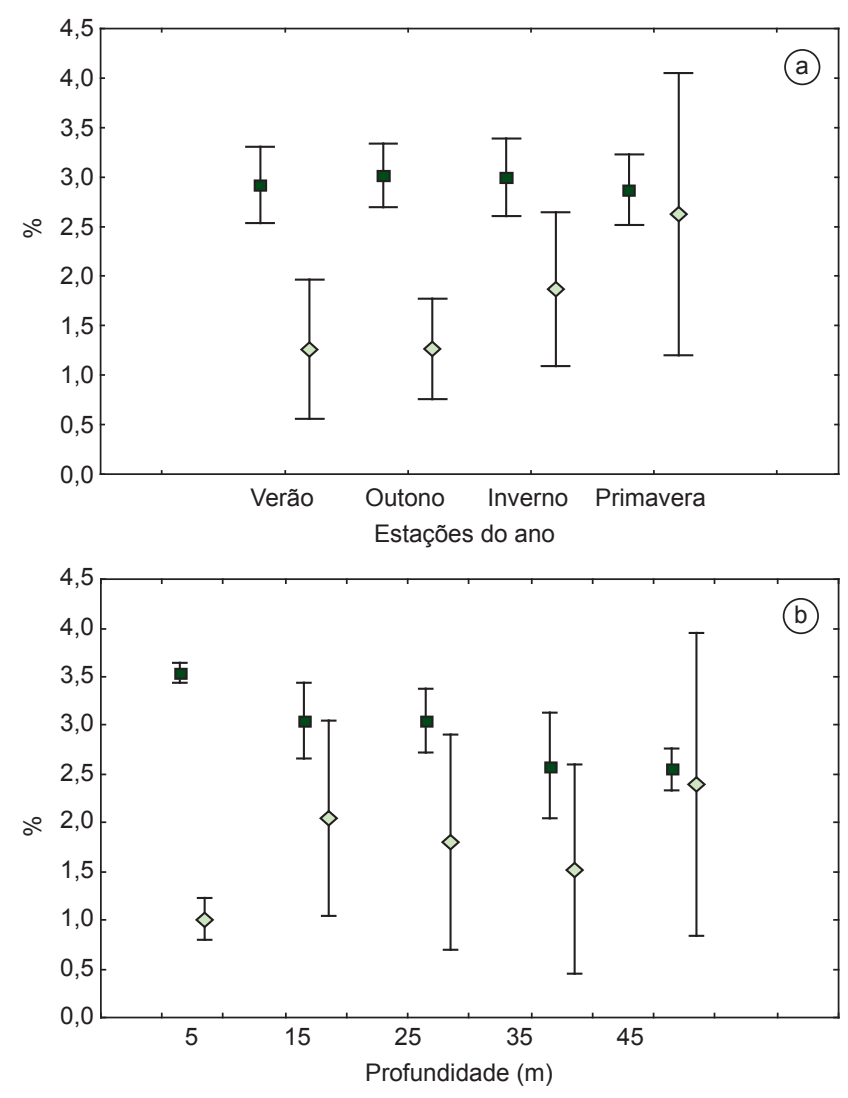

- phi $\diamond \mathrm{mo}$

Figura 4. Média e intervalo de confiança (95\%) do diâmetro médio do sedimento (phi) e do teor de matéria orgânica (MO) a) nas estações do ano de $2001 \mathrm{e}$ b) em diferentes profundidades.

Figure 4. Mean phi and organic matter contents and confidence interval (95\%) a) at 2001 seasons and b) at different depths.

lação significativa, porém negativa $(\mathrm{r}=-0,60, \mathrm{p}=0,007)$. Assim, esta análise exploratória resultou na retirada de Caraguatatuba no modelo final da ANCOVA (Tabela 3), que apresentou homogeneidade de variâncias $(p>0,05)$, normalidade dos resíduos $(p=0,063)$ e interação não significativa entre a covariável e as regiões $(\mathrm{p}=0,814)$. Como resultado, $47 \%$ da variabilidade na biomassa dos peixes Sciaenidae pode ser explicada pelo modelo, não havendo diferenças significativas entre as regiões de Ubatuba e São Sebastião (Tabela 3).

Os resultados da CCA mostraram que o camarão X. kroyeri foi o único fator importante na distribuição das espécies no eixo 1 . No eixo 2 Farfantepenaeus spp. e Litopenaeus schmitti foram os principais fatores atuando no gradiente de distribuição das espécies de peixes Sciaenidae. A primeira esteve relacionada com os transectos localizados nas profundidades 35 e 45 m enquanto a última, naqueles situados nos 5, 15 e 25 m (Tabela 4, Figura 6). A configuração dos pontos da CCA não foi dependente das estações do ano e das regiões (teste de Kruskal-Wallis, p > 0,05). A correlação de Spearman entre o eixo 2 da CCA e as profundidades foi positiva e significativa ( $r s=0,56, p=0,005$ ). Assim, a distribuição de Farfantepenaeus spp. e $L$. schmitti parece ser dependente do gradiente de profundidades amostradas. Os eixos 1 e 2 da CCA explicaram $86 \%$ da variabilidade dos dados. Com as análises de correlação de Spearman, foram verificadas correlações significativas entre Farfantepenaeus spp. e Ctenosciaena gracilicirrhus $(\mathrm{p}<0,05)$ e entre e L. schmitti e Paralonchurus brasiliensis $(\mathrm{p}<0,01)$.
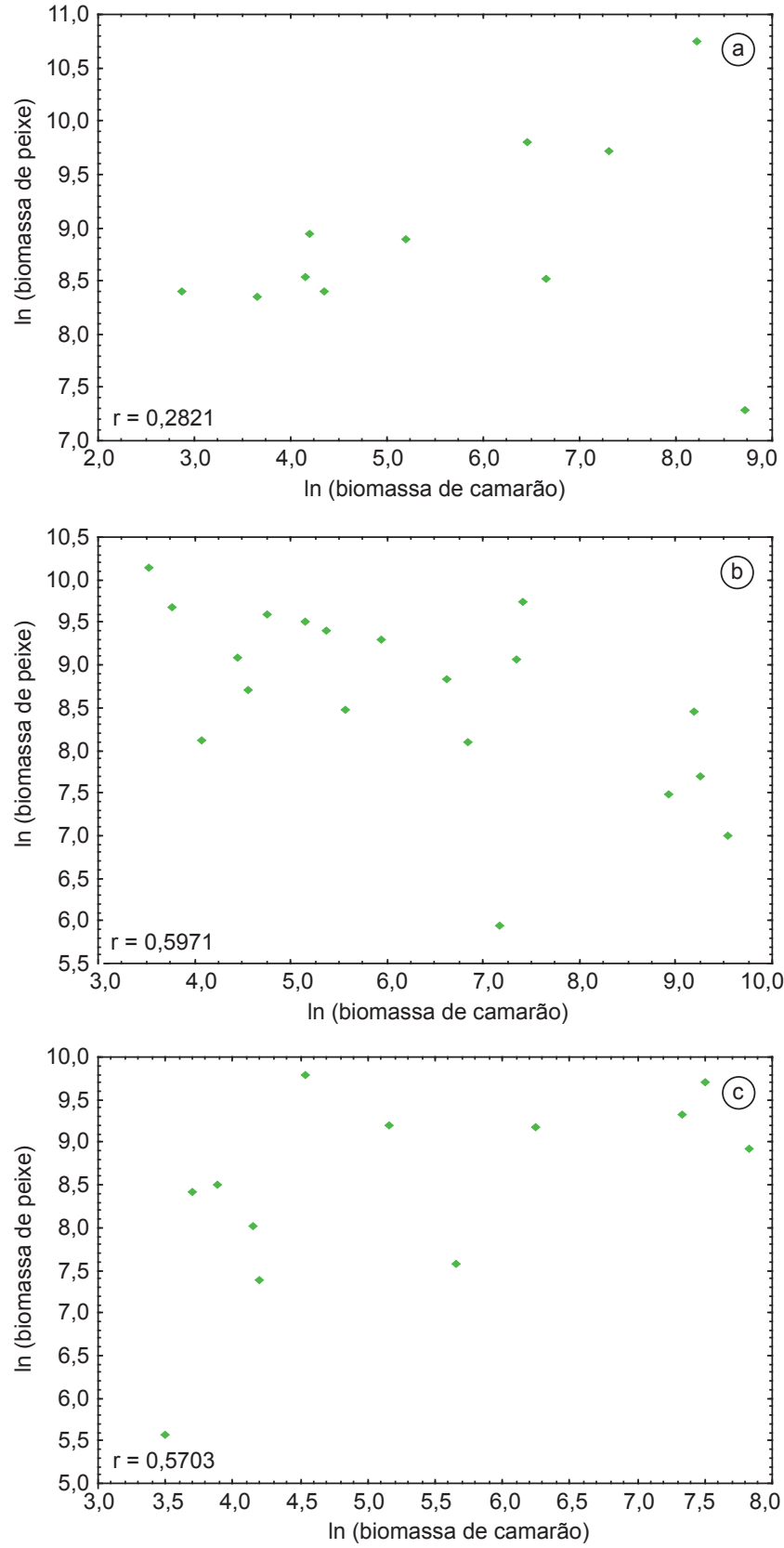

Figura 5. Correlações entre o logaritmo natural da biomassa de peixes e o logaritmo natural da biomassa de camarões, a) nas regiões de Ubatuba, b) Caraguatatuba e c) São Sebastião.

Figure 5. Correlations between the natural logarithm of fish biomass and the natural logarithm of shrimp biomass a) at Ubatuba, b) Caraguatatuba and c) São Sebastião.

\section{Discussão}

Na costa norte do estado de São Paulo existem três massas de água, com diferentes dinâmicas nas estações do ano: Água Costeira (AC), com alta temperatura e baixa salinidade $\left(\mathrm{T}>20{ }^{\circ} \mathrm{C}, \mathrm{S}<36\right.$ ); Água Tropical, com temperatura e salinidade elevadas $\left(\mathrm{T}>20^{\circ} \mathrm{C}\right.$, $\mathrm{S}>36$ ) e Água Central do Atlântico Sul (ACAS), com temperatura e salinidade baixas $\left(\mathrm{T}<18{ }^{\circ} \mathrm{C}, \mathrm{S}<36\right.$ ) (Castro Filho et al. 1987). A ACAS encontra-se sob a Corrente do Brasil em áreas oceânicas, penetrando sobre a plataforma continental durante a primavera e verão, formando uma termoclina, recuando no inverno para áreas mais 
Souza, U. P. et al.

Tabela 2. Biomassas (g) das espécies de peixes Sciaenidae e de camarões Penaeoidea amostradas em Ubatuba, Caraguatatuba e São Sebastião, durante o ano 2001

Table 2. Sciaenidae fish and Penaeoidea shrimp biomass (g) sampled at Ubatuba, Caraguatatuba and São Sebastião, during 2001.

\begin{tabular}{|c|c|c|c|}
\hline Sciaenidae & Biomassa & Penaeoidea & Biomassa \\
\hline Ctenosciaena gracilicirrhus (Metzelaar, 1919) & 63.436 & Penaeidae & \\
\hline Cynoscion guatucupa (Cuvier, 1830) & 14.794 & Artemesia longinaris Bate, 1888 & 2.484 \\
\hline Cynoscion jamaicensis (Vaillant \& Bocourt, 1833) & 22.287 & Farfantepenaeus brasiliensis (Latreille, 1817) & 4.749 \\
\hline Cynoscion virescens (Cuvier, 1830) & 1.057 & Farfantepenaeus paulensis (Pérez-Farfante, 1967) & 1.883 \\
\hline Isopisthus parvipinnis (Cuvier, 1830) & 6.164 & Litopenaeus schmitti (Burkenroad, 1936) & 4.748 \\
\hline Larimus breviceps Cuvier, 1830 & 34.508 & Rimapenaeus constrictus (Stimpson, 1874) & 1.163 \\
\hline Macrodon ancylodon (Bloch \& Schneider, 1801) & 4.069 & Xiphopenaeus kroyeri (Heller, 1862) & 48.093 \\
\hline Menticirrhus americanus (Linnaeus, 1758) & 47.417 & Solenoceridae & \\
\hline Micropogonias furnieri (Desmarest, 1823) & 49.519 & Pleoticus muelleri (Bate, 1888) & 15.586 \\
\hline Nebris microps Cuvier, 1830 & 123 & Sicyoniidae & \\
\hline Ophioscion punctatissimus Meek \& Hildebrand, 1925 & 11.719 & Sicyonia dorsalis Kingsley, 1878 & 240 \\
\hline Paralonchurus brasiliensis (Steindachner, 1875) & 81.278 & Sicyonia typica (Boeck, 1864) & 390 \\
\hline Stellifer brasiliensis (Schultz, 1945) & 3.134 & & \\
\hline Stellifer rastrifer (Jordan, 1889) & 11.512 & & \\
\hline Stellifer stellifer (Bloch, 1790) & 132 & & \\
\hline Umbrina canosai Berg, 1895 & 24.626 & & \\
\hline Umbrina coroides Cuvier, 1830 & 2.836 & & \\
\hline
\end{tabular}

Tabela 3. Resultados da Análise de covariância (ANCOVA). Variável dependente: logaritmo natural da biomassa de peixes. Covariável: logaritmo natural da biomassa de camarões. Variáveis categóricas: regiões de Ubatuba e São Sebastião $(\alpha<0,05)\left(n=22 ; r^{2}=0,466\right)$.

Table 3. Analysis of covariance (ANCOVA) results. Response variable: natural logarithm of fish biomass. Covariate: natural logarithm of shrimp biomass. Categorical factors: Ubatuba and São Sebastião areas $(\alpha<0,05)$ $\left(\mathrm{n}=22 ; \mathrm{r}^{2}=0,466\right)$.

\begin{tabular}{lcrrrc}
\hline Fontes de variação & SQ & gl & QM & F & P \\
\hline Camarões & 9,138 & 1 & 9,138 & 13,176 & $\mathbf{0 , 0 0 2}$ \\
Regiões & 0,325 & 1 & 0,325 & 0,468 & 0,503 \\
Camarões * Regiões & 0,039 & 1 & 0,039 & 0,057 & 0,814 \\
Resíduo & 12,483 & 18 & 0,694 & & \\
\hline
\end{tabular}

Tabela 4. Resultados da Análise de Correspondência Canônica (CCA) para a distribuição das espécies de Sciaenidae em relação aos camarões Penaeoidea, amostradas em Ubatuba e São Sebastião, durante o ano 2001.

Table 4. Results of canonical correspondence analysis (CCA) to Sciaenidae fish distribution in relation to Penaeoidea shrimp, sampled from Ubatuba and São Sebastião, during 2001.

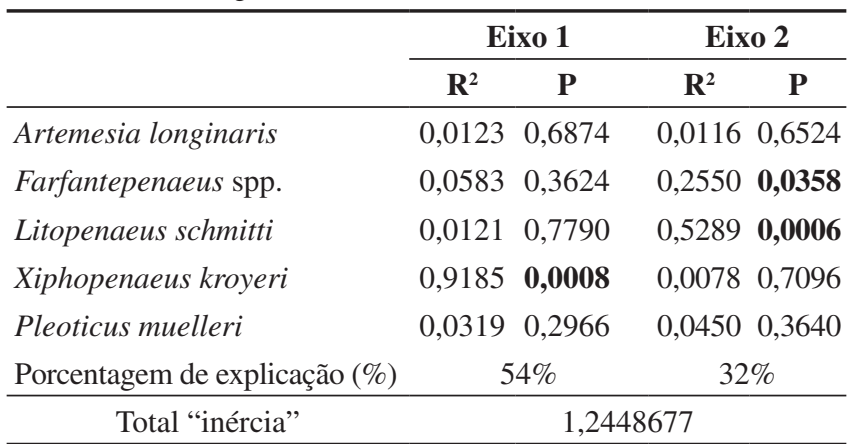

afastadas da costa (Matsuura 1986, Castro Filho et al. 1987). A movimentação da frente oceânica, do fundo à superfície, influencia tanto a estrutura oceanográfica, quanto a distribuição sazonal de organismos e a dinâmica do ecossistema da região (Pires-Vanin et al. 1993). No presente estudo, a penetração da ACAS ficou evidente na primavera e no verão, nas maiores profundidades, sendo também as estações do ano e os transectos onde foram verificadas as maiores diferenças entre as temperaturas de superfície e de fundo (Figura 2a, b). Pires-Vanin (1993), amostrando áreas próximas, encontrou uma penetração incipiente da ACAS na primavera, tornando-se mais acentuada no verão, quando a água fria aproximou-se da costa.

Verificou-se que as maiores biomassas dos peixes e dos camarões foram nos meses de inverno, nos transectos mais rasos. Nesta estação ocorrem passagens de frentes frias intensas, cujo efeito no ambiente marinho é provocar o revolvimento do fundo, especialmente na plataforma interna, que corresponde às menores profundidades (Pires-Vanin 1993). Além disso, no inverno o domínio interior é ocupado pela AC, com águas mais quentes e com menor salinidade (Castro Filho et al. 1987), características favoráveis ao estabelecimento de algumas espécies de camarões como Xiphopenaeus kroyeri, Pleoticus muelleri, Artemesia longinaris e Litopenaeus schmitti (Pires 1992).

As estações do ano influenciam o ritmo alimentar das espécies de peixes não-migrantes devido, principalmente, às diferenças de temperatura da água e à duração do dia e da noite, que determinam uma maior ou menor disponibilidade de alimento (Zavala-Camin 1996). No inverno, as fontes alimentares são principalmente bênticas, favorecendo mudanças tróficas, com conseqüente alteração dos padrões do fluxo de energia e matéria na plataforma continental da região de Ubatuba (Pires-Vanin et al. 1993). Assim, sugere-se que a retração da ACAS e a ampliação da área ocupada pela AC no inverno, em profundidades menores que $20 \mathrm{~m}$ resultando em temperaturas mais altas, favoreçam a presença da fauna bentônica de peixes e de camarões. A passagem de correntes frias e ocasionais, principalmen- 


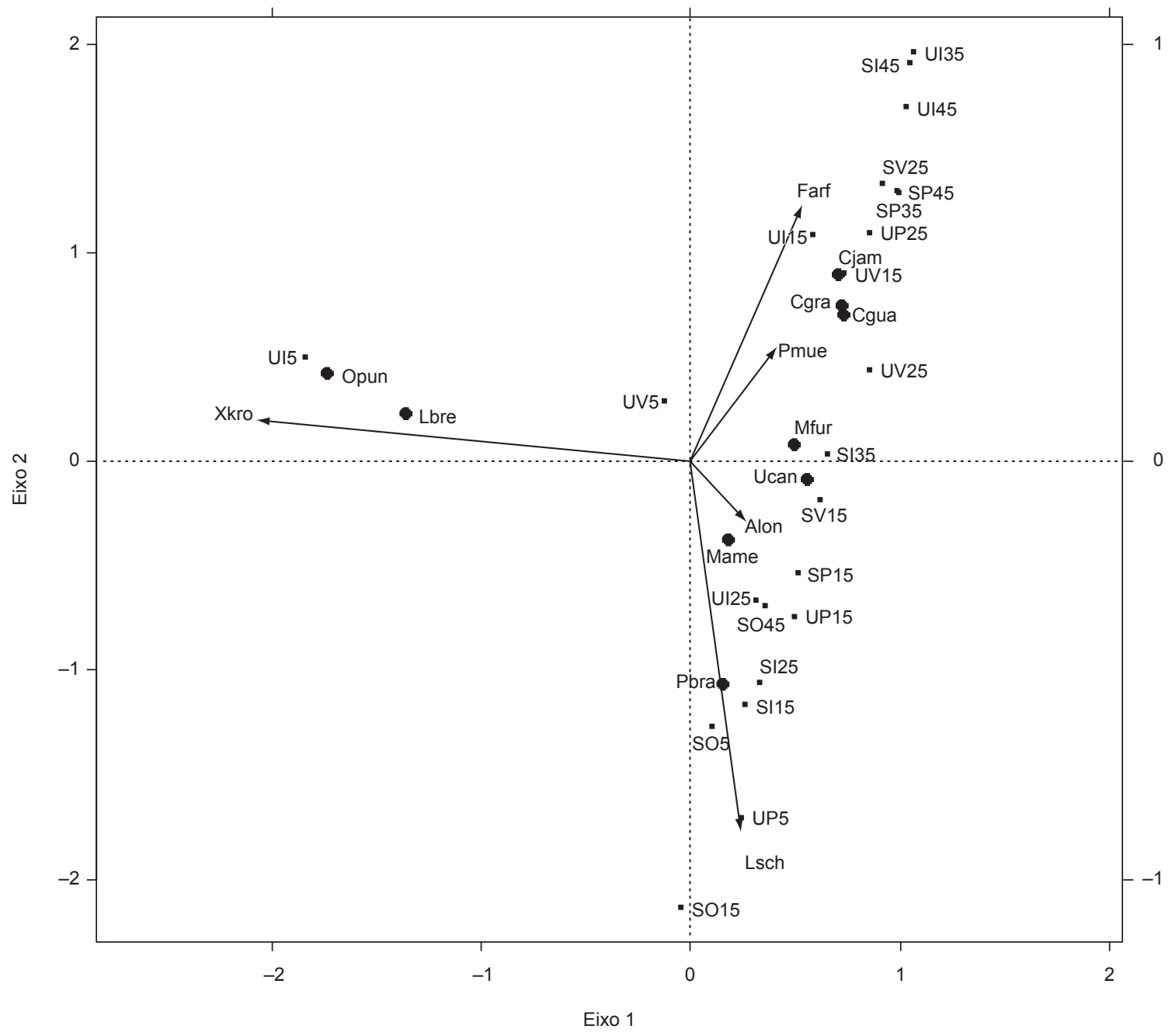

Figura 6. Resultados da Análise de Correspondência Canônica $(\mathrm{CCA})$ entre as espécies de camarões (Xkro = Xiphopenaeus kroyeri, Farf $=$ Farfantepenaeus spp., Pmue = Pleoticus muelleri, Alon = Artemesia longinaris e Lsch = Litopenaeus schmitti $)$ e as espécies de peixes $($ Opun = Ophioscion punctatissimus, Lbre $=$ Larimus breviceps, Cjam $=$ Cynoscion jamaicensis, Cgra $=$ Ctenosciaena gracilicirrhus, Cgua $=$ Cynoscion guatucupa, Mfur $=$ Micropogonias furnieri, Ucan = Umbrina canosai , Mame = Menticirrhus americanus e Pbra = Paralonchurus brasiliensis $)$, nas estações do ano de 2001 (Verão = V; Outono = O; Inverno = I e Primavera $=$ P) e nas profundidades amostradas $(5,15,25,35$ e 45 m) em Ubatuba (U) e em São Sebastião (S).

Figure 6. Results of Canonical Correspondence Analysis (CCA) between shrimp (Xkro = Xiphopenaeus kroyeri, Farf = Farfantepenaeus spp., Pmue $=$ Pleoticus muelleri, Alon = Artemesia longinaris, and Lsch $=$ Litopenaeus schmitti $)$ and fish $($ Opun $=$ Ophioscion punctatissimus, Lbre $=$ Larimus breviceps, Cjam $=$ Cynoscion jamaicensis, Cgra $=$ Ctenosciaena gracilicirrhus, Cgua $=$ Cynoscion guatucupa, Mfur = Micropogonias furnieri, Ucan $=$ Umbrina canosai, Mame $=$ Menticirrhus americanus, and Pbra $=$ Paralonchurus brasiliensis $)$, at 2001 seasons $($ Summer $=\mathrm{V} ;$ Autumm $=$ O; Winter $=\mathrm{I}$ and Spring $=\mathrm{P})$ and in depths $(5,15,25,35$ e $45 \mathrm{~m})$ at Ubatuba and São Sebastião.

te em locais mais rasos, torna o substrato mais instável, podendo expor as espécies de camarões que tenham o hábito de se enterrar à predação pelos peixes Sciaenidae, presentes em abundância nestas profundidades.

Foram encontrados valores elevados de phi nas áreas mais rasas das regiões, caracterizando um substrato composto por frações finas de sedimento, onde também foram amostradas as maiores biomassas de peixes (5 m) e de camarões (15 m). Segundo Vazzoler (1975), o tipo de substrato limita a distribuição e a abundância dos Sciaenidae e também da fauna de invertebrados bênticos. Para a maioria dos camarões Penaeoidea, a distribuição espacial das espécies é mais influenciada pela textura do substrato, principalmente em locais com sedimentos compostos por silte e argila (Costa \& Fransozo 2004, Costa et al. 2004, 2007). Desse modo, um substrato composto por sedimentos mais finos estaria favorecendo o estabelecimento e manutenção dos camarões no ambiente, contribuindo para as relações ecológicas com os Sciaenidae, já que a maioria das espécies amostradas ocorre preferencialmente em substratos arenosos e areno-lodosos.

A composição e a dominância específica das comunidades bênticas variam em todas as faixas de profundidades, confirmando a importância desta variável como um fator estruturador das comunidades na região de Ubatuba (Pires-Vanin 1993), corroborando os resultados registrados no presente estudo. Assim, a profundidade bem como a temperatura, sistemas de correntes, salinidade, oxigenação e disponibilidade de alimento, são os principais fatores na organização da fauna marinha (Lowe-McConnel 1999). Estudos como os de Fransozo et al. (2000), Costa \& Fransozo (2004) e Castro et al. (2004) têm demonstrado que os camarões de menores tamanhos habitam locais mais rasos, constituindo mais um fator que poderia influenciar os padrões de distribuição dos Sciaenidae, considerando que a predação provavelmente esteja direcionada a camarões menores e sugerindo que as regiões costeiras configuram ambientes propícios 
à alimentação e crescimento dos peixes, como mencionado por Braga \& Goitein (1984)

Os resultados indicaram que as regiões de Ubatuba e São Sebastião foram semelhantes, com correlações positivas e significativas entre as biomassas dos peixes e dos camarões. Os transectos amostrados nestas duas regiões encontram-se menos expostos e próximos a algumas ilhas, o que não ocorre nos transectos amostrados em Caraguatatuba (vide Figura 1). A presença do Canal e da Ilha de São Sebastião torna a região sul mais protegida, apresentando depósitos de sedimentos finos e elevado teor de matéria orgânica, além de ser uma região que sofre influência de águas quentes (Pires 1992). Os pontos selecionados para as amostragens em Ubatuba podem estar sob influências semelhantes, considerando que os transectos de 5 a $35 \mathrm{~m}$ situam-se próximos à Ilha das Couves e que as menores profundidades $(5$ e $15 \mathrm{~m}$ ) encontram-se em regiões mais abrigadas, próximas à linha da costa. Para animais bentônicos, como os caranguejos braquiúros, as ilhas são fundamentais para o aumento da riqueza e para o estabelecimento das espécies em enseadas na região de Ubatuba (Bertini et al. 2004). Em condições ambientais favoráveis ao estabelecimento e manutenção das espécies, as relações ecológicas entre os peixes e os camarões tornam-se mais prováveis, como evidenciado pela correlação entre suas biomassas, nas regiões de Ubatuba e São Sebastião.

Em Caraguatatuba as correlações entre as biomassas dos grupos estudados foram significativas, porém negativas e as biomassas totais muito elevadas. Este resultado pode estar relacionado à grande biomassa de Xiphopenaeus kroyeri, que correspondeu a 73\% do total de camarões amostrados nesta região. No entanto, os resultados revelaram que as relações entre os peixes e os camarões em Caraguatatuba foram distintas das observadas em Ubatuba e São Sebastião, sugerindo que diferentes fatores bióticos e abióticos podem determinar as relações das espécies nos ambientes. Estas observações corroboram o fato de que não existe uma única variável ambiental que controle as preferências ambientais dos camarões, mas um conjunto de fatores associados (Ye et al. 1999).

Os resultados da CCA mostraram que $X$. kroyeri representou a maior contribuição na distribuição das espécies no eixo 1, e que apresenta preferência por locais mais rasos, corroborando os resultados encontrados por Costa et al. (2007) na região de Ubatuba. Esta espécie é a mais explorada na costa do estado de São Paulo (Rodrigues et al. 1992, Nakagaki \& Negreiros-Fransozo 1998) e possui importância ecológica para as relações tróficas, contribuindo para a estabilidade de comunidades bênticas (Pires 1992). Apesar da representatividade de $X$. kroyeri no eixo 1 e da elevada biomassa em todas as regiões amostradas, não foram verificadas correlações significativas entre X. kroyeri e as espécies de Sciaenidae. Segundo Dall et al. (1990), os Penaeidae dispõem de mecanismos variados para evitar a predação, utilizando a vegetação subaquática, enterrando-se no substrato, vivendo em águas turvas, restringindo o período de atividade, formando grandes grupos e/ou tendo uma resposta de fuga bem desenvolvida. Fatores como a palatabilidade, o tamanho, a acessibilidade, a atividade e a densidade da presa e do predador, poderiam interferir no processo de predação (Pereira et al. 1983 apud Nakagaki 1999), considerando que muitas espécies de peixes são predadores visuais. Nakagaki (1999) sugeriu que, em seu ambiente natural, X. kroyeri apresenta movimentos rápidos, nadando continuamente. Este comportamento foi confirmado pelo mesmo autor em aquários, além da observação de que a espécie enterra-se parcialmente no substrato, quando em repouso, e que seu rostro pronunciado poderia evitar a seleção pelos potenciais predadores. Portanto, a ausência de correlação estatística entre os peixes e este camarão pode ter sido resultado das características morfológicas e comportamentais de ambos os grupos, relacionadas ao processo de busca e captura do alimento.
No eixo 2 os camarões Farfantepenaeus spp. e Litopenaeus schmitti foram as principais espécies que contribuíram para a distribuição dos peixes, com diferenças em relação às profundidades. Segundo Iwai (1978), Farfantepenaeus brasiliensis e F. paulensis permanecem enterrados durante o dia, emergindo apenas à noite, preferindo temperaturas elevadas $\left(>23{ }^{\circ} \mathrm{C}\right)$ e fundos arenosos (Williams 1984). Ctenosciaena gracilicirrhus foi uma das principais espécies de peixe amostradas, importante neste mesmo eixo, apresentando correlações significativas com Farfantepenaeus spp., mais abundante em transectos profundos ( 35 e $45 \mathrm{~m}$ ). Esta espécie ocorre preferencialmente em águas "offshore", em fundos de areia grossa e apresenta boca inferior com um barbilhão mentoniano, característica de peixes que se alimentam no fundo (Chao 1978, Cunningham 1989). A morfologia desta espécie e a preferência por locais mais profundos poderiam facilitar a busca e o encontro de itens no substrato por meio de vasculhamento, favorecendo uma interação com Farfantepenaeus spp.

Paralonchurus brasiliensis foi a principal espécie nas regiões de Caraguatatuba e São Sebastião, corroborando os resultados de Coelho et al. (1993), que verificaram a importância da mesma como fauna acompanhante na pesca do camarão sete-barbas (X. kroyeri) no litoral do estado de São Paulo. Vários autores verificaram que, apesar de $P$. brasiliensis apresentar o item Crustacea em sua dieta, sua alimentação é preferencialmente anelidófaga (Amaral \& Migotto 1980, Braga et al. 1985, Capitoli et al. 1994, Micheletti \& Uieda 1996, Jucá-Chagas 1997, Soares \& Vazzoler 2001). Apesar da alta preferência por Polychaeta, as correlações entre P. brasiliensis e L. schmitti foram significativas, estando as duas espécies representadas no segundo eixo da CCA e com influência dos transectos rasos $(5,15$ e $25 \mathrm{~m}$ ), locais onde também foram observados sedimentos mais finos. Paralonchurus brasiliensis pode ser caracterizada como uma espécie tropical, associada à águas quentes e a fundos arenosos e arenolodosos, ocorrendo em profundidades inferiores a $25 \mathrm{~m}$ (Menezes \& Figueiredo 1980, Rocha 1990). Da mesma forma, L. schmitti prefere temperaturas elevadas, sendo uma espécie típica de regiões tropicais (Costa 2002, Fransozo et al. 2002). As correlações entre P. brasiliensis e L. schmitti podem ter sido influenciadas por preferências individuais em relação às características dos ambientes.

Larimus breviceps foi a principal espécie amostrada na região de Ubatuba e a ausência de correlações com as espécies de camarões poderia ser explicada pelas características morfológicas da espécie, que apresenta boca com orientação oblíqua, rastros branquiais longos e numerosos (Chao 1986), facilitando a utilização de itens pelágicos em sua dieta, composta principalmente por camarões sergestídeos (Micheletti \& Uieda 1996, Lowe-McConnell 1999, Jucá-Chagas 1997, Soares \& Vazzoler 2001). Outras espécies abundantes foram Micropogonias furnieri e Menticirrhus americanus, classificadas por Chao (1978) como espécies que se alimentam no fundo, devido à boca inferior e à presença de barbilhões. A primeira apresenta pequenos tufos de barbilhões na mandíbula inferior e se alimenta de pequenos crustáceos e anelídeos (Menezes \& Figueiredo 1980, Micheletti \& Uieda 1996, Lowe-McConnell 1999, Soares \& Vazzoler 2001). A última apresenta um barbilhão mentoniano, alimentando-se de crustáceos e poliquetos no fundo (Menezes \& Figueiredo 1980, Jucá-Chagas 1997, Rondineli et al. no prelo).

Apesar de terem sido verificadas poucas correlações significativas, estudos de alimentação têm confirmado a importância de determinadas espécies de camarões para os Sciaenidae, como, por exemplo, de Artemesia longinaris para Macrodon ancylodon, Menticirrhus americanus (Juras \& Yamaguti 1985, Capitoli et al. 1994), Cynoscion guatucupa (Cazorla 1996), Umbrina canosai (Haimovici et al. 1989) e Stellifer rastrifer (UP. Souza, dados não publicados); de Pleoticus muelleri para Umbrina canosai (Haimovici et al. 1989) e 
de Xiphopenaeus kroyeri para Cynoscion virescens, Nebris microps (Rodrigues \& Meira 1988) e Stellifer rastrifer (Camargo \& Isaac 2004, U.P. Souza, dados não publicados).

Segundo Chao \& Musick (1977), entre os peixes da família Sciaenidae a coexistência de espécies pode ser atribuída a diferenças temporais em suas distribuições, à abundância de recursos alimentares nas áreas ocupadas e a variações nas adaptações ao hábitat e ao hábito alimentar. Em geral, estas diferenças são acompanhadas de características morfológicas que possibilitam a utilização de recursos de forma diferenciada, evitando a sobreposição alimentar. A soma de características como diversidade e tamanho de itens consumidos, morfologia do predador e posição que a presa ocupa na coluna d'água, reduzem a sobreposição alimentar de peixes Sciaenidae, determinando diferenças de comportamento que permitem a coexistência das espécies (Micheletti \& Uieda 1996, Jucá-Chagas 1997).

Dadas as diversas relações ecológicas conhecidas na literatura entre os peixes Sciaenidae e os camarões Penaeoidea, é possível que muitas espécies de peixes busquem as mesmas áreas de ocorrência dos camarões em função dos fatores abióticos ou para utilizá-los como recurso alimentar. Os resultados deste estudo sugerem que o tipo de sedimento e a profundidade foram os fatores preponderantes nas distribuições espacial e temporal dos Sciaenidae nas regiões de Ubatuba, Caraguatatuba e São Sebastião. Estes fatores agem de forma complexa sobre a abundância destas espécies de peixes, assim como sobre os camarões, podendo ser determinantes na ocorrência e intensidade das relações ecológicas entre estes grupos.

\section{Agradecimentos}

À FAPESP (Fundação de Amparo à Pesquisa do Estado de São Paulo) pelo apoio logístico mediante o Projeto BIOTA (\#98/07090-3). A autora (U.P.S) agradece à CAPES (Coordenadoria de Aperfeiçoamento de Pessoal de Nível Superior) pela bolsa concedida no mestrado, a todos os membros do grupo NEBECC que ajudaram nos trabalhos de campo, ao MsC. Fabio Cop Ferreira pelo auxílio nas análises e revisão do manuscrito e ao desenhista Jaime R. Somera pela confecção do mapa.

\section{Referências Bibliográficas}

AMARAL, A.C.Z. \& MIGOTTO, A.E. 1980. Importância dos anelídeos poliquetas na alimentação da macrofauna demersal e epibentônica da região de Ubatuba. Bolm Inst. oceanogr. 29(2):31-35.

BERTINI, G., FRANSOZO, A. \& MELO, G.A.S. 2004. Biodiversity of brachyuran crabs (Crustacea: Decapoda) from non-consolidated sublittoral bottom on the northern coast of São Paulo State, Brazil. Biodivers. Conserv. 13:2185-2207.

BRAGA, F.M.S. \& GOITEIN, R. 1984. Lista prévia das espécies de peixes demersais na região da Ilha Anchieta (Lat. $23^{\circ} 33^{\prime}$ S, Long. $45^{\circ} 05^{\prime} \mathrm{W}$ ), Ubatuba, litoral norte do Estado de São Paulo, Brasil. Naturalia 9:61-72.

BRAGA, F.M.S., BRAGA, M.A.A.S. \& GOITEIN, R. 1985. Fator de condição e alimentação de Paralonchurus brasiliensis (Osteichthyes, Sciaenidae) na região da Ilha Anchieta (Lat. $23^{\circ} 33^{\prime}$ S-Long. $45^{\circ} 05^{\prime}$ W) Ubatuba, Estado de São Paulo. Naturalia 10:1-11.

BREWER, D.T., BLABER, S.J.M., SALINI, J.P. \& FARMER, M.J. 1995. Feeding ecology of predatory fishes from Groote Eylandt in the Gulf of Carpentaria Australia, with special reference to predation on Penaeid prawns. Estuar. Coast Shelf S. 40:577-600.

CAMARGO, M. \& ISAAC, V. 2004. Food categories reconstruction and feeding consumption estimates for the Sciaenid Macrodon ancylodon (Bloch \& Schneider), and the congeneric fishes Stellifer rastrifer (Jordan) and Stellifer naso (Jordan) (Pisces, Perciformes) in the Caeté Estuary, Northern Coast of Brazil. Rev. Bras. Zool. 21(1):85-89.
CAPITOLI, R.R., BAGER, A. \& RUFFINO, M.L. 1994. Contribuição ao conhecimento das relações tróficas bentônico-demersais nos fundos de pesca do camarão Artemesia longinaris Bate, na região da Barra da Lagoa dos Patos, RS, Brasil. Nauplius 2:53-74.

CASTRO, R.H., COSTA, R.C., FRANSOZO, A. \& MANTELATTO, F.L. 2004. Population structure of the seabob shrimp Xiphopenaeus kroyeri (Heller, 1862) (Crustacea: Penaeoidea) in the littoral of São Paulo. Sci. Mar. 69(1):1-8

CASTRO FILHO, B.M., MIRANDA, L.B. \& MIYAO, S.Y. 1987. Condições oceanográficas na plataforma continental ao largo de Ubatuba: variações sazonais e em média escala. Bolm Inst. oceanogr. 35(2):135-151.

CAZORLA, A.L. 1996. The food of Cynoscion striatus (Cuvier) (Pisces: Sciaenidae) in the Bahía Blanca area, Argentina. Fish. Res. 28:371-179.

CERVIGÓN, F., CIPRIANI, R., FISHER, W., GARIBALDI, L., HENDRICKX, M., LEMUS, A.J., MÁRQUEZ, R., POUTIERS, J.M., ROBAINA, G. \& RODRIGUEZ, B. 1992. Field guide to the comercial marine and brackishwater resources of the Northern Coast of South America. FAO, Rome.

CHAO, L.N. 1978. A basis for classifying western Atlantic Sciaenidae (Teleostei: Perciformes). NOAA Tech. Rep. 415:1-64.

CHAO, L.N. 1986. A synopsis on zoogeography of the Sciaenidae. In IndoPacific fish biology: Proceedings of the Second International Conference on Indo-Pacific Fishes (T. Uyeno, R. Arai, T. Taniuchi \& K. Matsuura, eds). Ichthyological Society of Japan, Tokyo, p.570-589.

CHAO, L.N. \& MUSICK, J.A. 1977. Life history, feeding habits, and functional morphology of juvenile Sciaenid fishes in the York River, Virginia. Fish. Bull. 75(4):657-702.

COELHO, J.A.P., GRAÇA-LOPES, R., RODRIGUES, E.S., PUZZI, A. \& FARIAS, D.S. 1993. Aspectos biológicos e pesqueiros do Sciaenidae Paralonchurus brasiliensis (Steindachner, 1875) presente no rejeitado da pesca artesanal dirigida ao camarão sete-barbas (São Paulo, Brasil). Bol. Inst. Pesca. 20(único):95-101.

COSTA, R.C. 2002. Biologia e distribuição ecológica das espécies de camarões Dendrobranchiata (Crustacea: Decapoda) na região de Ubatuba (SP). Tese de doutorado, Universidade Estadual Paulista, Botucatu.

COSTA, R.C. \& FRANSOZO, A. 2004. Abundance and ecological distribution of the shrimp Rimapenaeus constrictus (Crustacea: Penaeidae) in the northern coast of São Paulo, Brazil. J. Nat. Hist. 38(7):901-912.

COSTA, R.C., FRANSOZO, A. \& PINHEIRO, A.P. 2004. Ecological distribution of the shrimp Pleoticus muelleri (Bate, 1888) (Decapoda: Penaeoidea) in southeastern Brazil. Hydrobiol. 529:195-203.

COSTA, R.C., FRANSOZO, A., FREIRE, F.A.M. \& CASTILHO, A.L. 2007. Abundance and ecologic distribution of the shrimp Xiphopenaeus kroyeri (Heller, 1862) (Decapoda, Penaeidae) in the northern Coast of São Paulo State, Brazil.region, SP, Brazil. Gulf and Caribbean Res. 19:33-41.

COSTA, R.C., FRANSOZO, A., MANTELATTO, F.L.M. \& CASTRO, R.H. 2000. Occurrence of shrimp species (Crustacea: Decapoda: Natantia: Penaeidae and Caridea) in Ubatuba Bay, Ubatuba, SP, Brazil. Proc. Biol. Soc. Wash. 113(3):776-781.

COSTA, R.C., FRANSOZO, A., SCHMIDT, G.A. \& FREIRE, F.A.M. 2003. Chave ilustrada para identificação dos camarões Dendrobranchiata do litoral norte do Estado de São Paulo. Biota Neotrop. 3(1): http://www. biotaneotropica.org.br/v3n1/pt/abstract?article+BN01503012003 (último acesso em 10/10/2007)

CUNNINGHAM, P.T.M. 1983. Estudo comparativo da ictiofauna da costa oeste e enseada das Palmas da Ilha Anchieta, enseada do Flamengo e enseada da Fortaleza (Lat. $23^{\circ} 29^{`} \mathrm{~S}-23^{\circ} 33^{`} \mathrm{~S}$, Long. $45^{\circ} 03^{`} \mathrm{~W}-45^{\circ} 09^{`} \mathrm{~W}$ ), Ubatuba, Estado de São Paulo, Brasil. Tese de doutorado, Universidade de São Paulo, São Paulo.

CUNNINGHAM, P.T.M. 1989. Observações sobre o espectro alimentar de Ctenosciaena gracilicirrhus (Metzellar), Sciaenidae. Rev. Bras. Biol. 49(2):335-339.

D'INCAO, F., VALENTINI, H. \& RODRIGUES, L.R. 2002. Avaliação da pesca de camarões nas regiões sudeste e sul do Brasil. Atlântica 24:103-116. 
DALL, W., HILL, B.J., ROTHLISBERG, P.C. \& STALPES, D.J. 1990. The biology of the Penaeidae. In Advances in marine biology (J.H.S. Blaxter \& A.J. Southward, eds). Academic Press, San Diego, v.27, p.283-355.

FRANSOZO, A., COSTA, R.C., PINHEIRO, M.A.A., SANTOS, S. \& MANTELATTO, F.L.M. 2000. Juvenile recruitment of the seabob Xiphopenaeus kroyeri (Heller, 1862) (Decapoda, Penaeidea) in the Fortaleza bay, Ubatuba, SP, Brazil. Nauplius 8(2):179-184.

FRANSOZO, A., COSTA, R.C., MANTELATTO, F.L.M., PINHEIRO, M.A.A. \& SANTOS, S. 2002. Composition and abundance of shrimp species (Penaeidea and Caridea) in Fortaleza bay, Ubatuba, São Paulo, Brazil. In Modern approaches study Crustacea (E. Escobar-Briones \& F. Alvarez, eds). Kluwer Academic/Plenum Publishers, p.117-123.

HAIMOVICI, M., TEIXEIRA, R.L. \& ARRUDA, M. DE C. 1989. Alimentação da castanha Umbrina canosai (Pisces: Sciaenidae) no sul do Brasil. Rev. Bras. Biol. 49(2):511-522.

HAKANSON, L. \& JANSSON, M. 1983. Principles of lake sedimentology. Springer-Verlag, Berlim

IWAI, M. 1978. Desenvolvimento larval e pós-larval de Penaeus (Melicertus) paulensis Pérez Farfante, 1967 (Crustacea, Decapoda) e o ciclo de vida dos camarões do gênero Penaeus da região Centro-Sul do Brasil. Tese de doutorado, Universidade de São Paulo, São Paulo.

JUCÁ-CHAGAS, R. 1997. Morfologia funcional relacionada à alimentação em Sciaenidae do litoral do Estado de São Paulo. Tese de doutorado, Universidade Estadual Paulista, Rio Claro.

JURAS, A.A. \& YAMAGUTI, N. 1985. Food and feeding habits of king weakfish, Macrodon ancylodon (Bloch \& Scheider, 1801) caught in the southern coast of Brazil (Lat. $29^{\circ}$ to $32^{\circ} \mathrm{S}$ ). Bolm Inst. oceanogr. 33(2):149-157.

LOWE-McCONNELL, R.H. 1999. Estudos ecológicos de comunidades de peixes tropicais (A.E.A.M. Vazzoler, A.A. Agostinho \& P.T.M. Cunningham, trad.). Editora da Universidade de São Paulo, São Paulo.

MACIA, A., ABRANTES, K.G.S. \& PAULA, J. 2003. Thorn fish Terapon jarbua (Forskal) predation on juvenile white shrimp Penaeus indicus $\mathrm{H}$. Milne Edwards and brown shrimp Metapenaeus monoceros (Fabricius): the effects of turbidity, prey density, substrate type and pneumatophore density. J. Exp. Mar. Biol. Ecol. 4142:1-28.

MATSUURA, Y. 1986. Contribuição ao estudo da estrutura oceanográfica da região sudeste entre Cabo Frio (RJ) e Cabo de Santa Marta Grande (SC). Ciênc. Cult. 38(8):1439-1450.

MENEZES, N.A. \& FIGUEIREDO, J.L. 1980. Manual de peixes marinhos do Sudeste do Brasil, IV. Teleostei (3). Museu de Zoologia da Universidade de São Paulo, São Paulo.

MENEZES, N.A., BUCKUP, P.A., FIGUEIREDO, J.L. \& MOURA, R.L. 2003. Catálogo das espécies de peixes marinhos do Brasil. Museu de Zoologia da Universidade de São Paulo, São Paulo.

MICHELETTI, C.V. \& UIEDA, V.S. 1996. Food resources partitioning among Sciaenid fishes (Perciformes: Sciaenidae) of the Flamengo bay, Ubatuba, southeastern Brazil. Arq. Biol. Tecnol. 39(3):639-649.

NAKAGAKI, J.M. 1999. Estudo da predação sobre camarões peneídeos (Crustacea, Decapoda). Tese de doutorado, Universidade Estadual Paulista, Botucatu.

NAKAGAKI, J.M. \& NEGREIROS-FRANSOZO, M.L. 1998. Population biology of Xiphopenaeus kroyeri (Heller, 1862) (Decapoda: Penaeidae) from Ubatuba bay, São Paulo, Brazil. J. Shellfish Res. 17(4):931-935.

NONATO, E.F., AMARAL, A.C.Z. \& FIGUEIREDO, J.L. 1983. Contribuição ao conhecimento da fauna de peixes do litoral norte do Estado de São Paulo. Bolm Inst. oceanogr. 32(2):143-152.

OKSANEN, J., KINDT, R., LEGENDRE, P. \& O'HARA, R.B. 2006. Vegan: community ecology package. Versão 1.8-3. http://www.r-project.org/ (último acesso em 01/10/2007)

PÉREZ-FARFANTE, I. \& KENSLEY, B. 1997. Penaeoid and Sergestoid shrimp and prawns of the World. Keys and diagnoses for the families and genera. Mémories du Muséum National d’Historie Naturalle, Paris.
PIRES, A.M.S. 1992. Structure and dynamics of benthic megafauna on the continental shelf offshore of Ubatuba, southeastern Brazil. Mar. Ecol. Prog. Ser. 86:63-76.

PIRES-VANIN, A.M.S. 1993. A macrofauna bêntica da plataforma continental ao largo de Ubatuba, São Paulo, Brasil. Publ. Espec. Inst. Oceanogr. (10): $137-158$.

PIRES-VANIM, A.M.S., ROSSI-WONGTSCHOWSKI, C.L.D.B., AIDAR, E., MESQUITA, H.S.L., SOARES, L.S.H., KATSURAGAWA, M. \& MATSUURA, Y. 1993. Estrutura e função do ecossistema de plataforma continental do Atlântico Sul brasileiro: síntese dos resultados. Publ. Espec. Inst. Oceanogr. (10):217-231.

R DEVELOPMENT CORE TEAM. 2007. R: a language and environment for statistical computing. Versão 2.4.1. Vienna, Austria, R Foundation for statistical computing. http://www.r-project.org/ (último acesso em 01/10/2007)

ROCHA, G.R.A. 1990. Distribuição, abundância e diversidade da ictiofauna

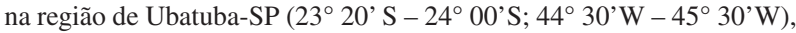
Brasil. Dissertação de mestrado, Universidade de São Paulo, São Paulo.

ROCHA, G.R.A. \& ROSSI-WONGTSCHOWSKI, C.L.D.B. 1998. Demersal fish community on the inner shelf of Ubatuba, South-eastern Brazil. Rev. Bras. Oceanogr. 46(2):93-109.

RODRIGUES, E.S. \& MEIRA, P. DE T.F. 1988. Dieta alimentar de peixes presentes na pesca dirigida ao camarão sete-barbas (Xiphopenaeus kroyeri) na Baía de Santos e praia de Perequê, Estado de São Paulo, Brasil. Bol. Inst. Pesca. 15(2):135-146.

RODRIGUES, E.S., PITA, J.B., GRAÇA LOPES, R., COELHO, J.A.P. \& PUZZI, A. 1992. Aspectos biológicos e pesqueiros do camarão sete-barbas (Xiphopenaeus kroyeri) capturados pela pesca artesanal no litoral do Estado de São Paulo. Bol. Inst. Pesca. 19 67-81.

RONDINELI, G.R., BRAGA, F.M.S., TUTUI, S.L.S. \& BASTOS, G.C.A. no prelo. Dieta de Menticirrhus americanus (Linnaeus, 1758) e Cynoscion jamaicensis (Vaillant \& Bocourt, 1883) (Pisces, Sciaenidae) no sudeste do Brasil, Estado de São Paulo. Bol. Inst. Pesca.

SHERIDAN, P.F., BROWDER, J.A. \& POWERS, J.E. 1984. Interactions with other species: ecological interactions between penaeid shrimp and bottomfish assemblages. In Penaeid shrimps - their biology and management (J.A. Gulland \& B.J. Rothschild, eds). Fishing New Books, Oxford, p.235-253.

SOARES, L.S.H. \& VAZZOLER, A.E.A.M. 2001. Diel changes in food and feeding activity of Sciaenid fishes from the South-Western Atlantic, Brazil. Rev. Bras. Biol. 61(2): 197-216.

TER BRAAK, C.J.F. 1986. Canonical Correspondence Analysis: a new eigenvector technique for multivariate direct gradient analysis. Ecology 67(5):1167-1179.

TUCKER, M. 1988. Techniques in sedimentology. Blackwell Sciaentific Publications, Oxford, p.1-394.

VAZZOLER, G. 1975. Distribuição da fauna de peixes demersais e ecologia dos Sciaenidae da plataforma continental brasileira, entre as latitudes $29^{\circ} 21^{\prime} \mathrm{S}$ (Tôrres) e 33 41' S (Chuí). Bolm Inst. oceanogr. 24:85-169.

WILLIAMS, A.B. 1984. Shrimps, lobsters and crabs of the Atlantic Coast of the Eastern United States, Maine to Florida. Smithsonian Institution Press, Washington.

YE, Y., MOHAMMED, H.M.A. \& BISHOP, J.M. 1999. Depth, temperature and salinity preferences of newly recruited shrimps in Kuwait waters. Fish. Oceanogr. 8(2):128-138.

ZAR, J.H. 1999. Bioestatistical analysis. 4 ed. Prentice Hall, New Jersey.

ZAVALA-CAMIN, L.A. 1996. Introdução aos estudos sobre alimentação natural em peixes. EDUEM, Maringá.

Recebido em 09/11/07 Versão reformulada recebida em 25/01/07 Publicado em 11/02/08 